\title{
Genomic data for personalized cancer medicine
}

\section{Introduction}

Personalized cancer care is not a new strategy; decisions regarding a patient's treatment were made taking into account the individual's disease and personal circumstances. However, the extent to which treatment can be specifically tailored has been limited. Most patients with a specific type and stage of cancer received the same treatment and it became clear that some treatments worked better for some patients. Advances in technology and the understanding of cancer biology are leading to more detailed descriptions of a patient's cancer and better targeted treatments. Such treatments limit damage to healthy tissue. The evolving field of personalized medicine is playing an increasingly important role in cancer prevention, diagnosis, prognosis and therapeutics.

\section{Cancer biology contribution}

It is now clear that tumors derived from the same organ can differ in extremely important ways. One step forward is represented by the possibility to classify cancers based on critical molecular targets. Drugs specifically acting against molecular targets in cancer cells -called targeted therapies- have been developed and are used to counteract some types of cancer in selected patients. It is so essential to pursue efforts in cancer research and gather comprehensive information on each tumor in order to be able to identify all involved targets and hence determine the most appropriate treatment for each tumor and patient.

The human genetic code is often the clue to health and to disease. Knowing that oncogenes are the key, there can be no doubt that genebased prevention and therapy will be crucial in winning the war on cancer. Now, things are changing and advances in technology and the results of the Human Genome Project have enabled researchers to identify the molecular features of each single tumor. Researchers have found that there is a wide heterogeneity among apparently similar tumors. One of the major discoveries of the last decade has been the identification of some specific alterations that are able to change the signal in the tumor cell and create a phenomenon called "addiction". This phenomenon means that the tumor can survive and reproduce itself mainly because this alteration is present. These alterations may be of different types. The majority of them are gene mutations, translocations and amplifications. It is therefore intuitive that giving a drug that targets these specific alterations is fundamental in achieving valuable improvements in competence performance and patient outcomes. This is the biological basis of personalized medicine.

Numerous recent studies have demonstrated the use of genomic data, particularly gene expression signatures. These studies highlight the opportunity for strategies to achieve truly personalized cancer treatment. Developing gene expression signatures will likely allow us to guide the use of currently available cancer drugs and elaborate new targeted therapeutics.

The practice of oncology continually faces the challenge of matching the right therapeutic regimen with the right patient; needless to say, this challenge is daunting, and, in present practice, we are often disappointed by suboptimal outcomes. These regimens do have specificity, not necessarily for a biologic target but rather
Volume 6 Issue 3 - 2016

\author{
Haji Adel Anis \\ Cedars-Jebel Ali International Hospital, UAE
}

Correspondence: Hajj Adel Anis, Medical Oncologist at Cedars-Jebel Ali International Hospital, 9370 Rue Lajeunesse, Montreal, UAE, Tel 438-992-55I6, Email ahaji@dr.com

Received: November 27, 2016 | Published: December 16, 2016

for a particular class of tumors. Gene expression patterns can help define specific tumor classes in a way that correlates directly with chemotherapeutic options. Even many targeted therapeutics, developed to specifically inhibit a biologic target activity, also lack a basis for selecting those patients who might benefit from the drug. Indeed there are some exceptions like Trastuzumab, Imatinib and various hormonal therapies. One approach to targeting biologic therapies is to employ gene expression data that predict sensitivity to a new therapeutic agent, similar to the strategy with the chemotherapies. Such an approach is developed by treating a series of cancer cell lines with the drug, measuring sensitivity on the basis of proliferation assays, and then using baseline expression data to develop the signature.

An alternative strategy makes use of gene expression patterns reflecting deregulation of oncogenic pathways. For instance, the role of alterations in Ras, Myc and the retinoblastoma protein in contributing to the development of oncogenic cells. Importantly, gene expression profiles provide a way to measure the consequences of the oncogenic process, irrespective of which specific aspect of the pathway is altered. The analysis of oncogenic pathways through gene expression analysis offers a potential opportunity to identify new therapeutic options for these patients. Pathway predictors provide a more efficient mechanism over predictors of response to each potential therapeutic agent. One major value of this approach is the capacity to direct combinations of therapies using multiple drugs that target multiple pathways on the basis of genomic information that describes the state of activity of the pathways.

Beyond the individual signatures assayed to date, an additional valuable resource is the availability of a database of signature information. These data provide a resource for evaluating opportunities for identifying patient subsets that may be appropriate for utilization of a given therapeutic in combination with a signature. This also provides the important additional opportunity of looking for appropriate combinations on the basis of patterns of these profiles.

\section{Conclusion}

Gene expression has recently been at the forefront of advance in personalized medicine. The ability to develop gene expression signatures will likely allow us to guide the use of currently available cancer drugs, develop new targeted therapeutics and provide an 
opportunity to better match the most effective drug or drugs with the molecular characteristics of the individual patient. Beyond the individual signatures assayed to date, an additional valuable resource is the availability of a database of signature information. This information serves not only as a guide to the identification of populations of patients that might be appropriate for a given therapeutic but can also identify potential combinations of drugs that might be most efficacious given the overlap in predicted sensitivities.

\section{Acknowledgments}

None.

\section{Conflicts of interest}

Author declares there are no conflicts of interest. 